\title{
Ludwig-Soret effect of aqueous solutions of ethylene glycol oligomers, crown ethers, and glycerol; temperature, molecular weight, and hydrogen bond effect
}

\author{
Kousaku Maeda, ${ }^{1}$ Naoki Shinyashiki, ${ }^{1}$ Shin Yagihara, ${ }^{1}$ Simone Wiegand, ${ }^{2}$ and Rio Kita ${ }^{1, a)}$ \\ ${ }^{1)}$ Department of Physics, Tokai University, Hiratsuka, Kanagwa 259-1292, Japan \\ ${ }^{2)}$ ICS-3 Soft Condensed Matter, Forschungszentrum Jülich GmbH, D-52428 Jülich, Germany, \\ and Department für Chemie - Physikalische Chemie, Universität zu Köln, 50939 Cologne, \\ Germany
}

(Dated: 2 September 2015)

\begin{abstract}
The thermal diffusion, also called the Ludwig-Soret effect, of aqueous solutions of ethylene glycol oligomers, crown ethers, and glycerol are investigated as a function of temperature by Thermal Diffusion Forced Rayleigh Scattering (TDFRS). The Soret coefficient, $S_{\mathrm{T}}$, and the thermal diffusion coefficient, $D_{\mathrm{T}}$, show a linear temperature dependence for all studied compounds in the investigated temperature range. The magnitudes and the slopes of $S_{\mathrm{T}}$ and $D_{\mathrm{T}}$ vary with the chemical structure of the solute molecules. All studied molecules contain ether and/or hydroxyl groups, which can act as acceptor or donor to form hydrogen bonds, respectively. By introducing the number of donor and acceptor sites of each solute molecule, we can express their hydrogen bond capability. $S_{\mathrm{T}}$ and $D_{\mathrm{T}}$ can be described by an empirical equation depending on the difference of donor minus acceptors sites and the molecular weight of the solute molecule.
\end{abstract}

PACS numbers: 61.25.he, 66.10.cd, 83.80.Rs

\section{INTRODUCTION}

Under a stable temperature gradient, $\nabla T$, molecules in a fluid mixture migrate along the temperature gradient. This effect is called thermal diffusion, thermodiffusion, or thermophoresis. For a binary mixture, the flux of component $1, \mathbf{J}_{1}$, can be expressed as $\mathbf{J}_{1}=$ $-\rho D \nabla w-\rho D_{\mathrm{T}} w(1-w) \nabla T$, where $\rho$ is the density of the mixture, $w$ is the weight fraction of component $1, D$ is the mutual diffusion coefficient, and $D_{\mathrm{T}}$ is the thermal diffusion coefficient ${ }^{1}$. In the steady state, with $\mathbf{J}_{1}=0$, the thermal diffusion and the Fick's diffusion balance, and the established concentration gradient, $\nabla w$, is characterized by the ratio of $D_{\mathrm{T}}$ to $D$ as follows,

$$
S_{\mathrm{T}} \equiv \frac{D_{\mathrm{T}}}{D}=-\frac{1}{w(1-w)} \frac{|\nabla w|}{|\nabla T|}
$$

Here, $S_{\mathrm{T}}$ is the Soret coefficient ${ }^{2,3}$.

One of the important applications of the thermodiffusion nowadays is the determination of binding constants of biochemical reactions ${ }^{4}$. Although, the process is not understood on a microscopic level, especially in aqueous systems the effect is often accompanied by changes in the hydration shell of the solute molecules, which can lead to significant changes of the thermophoretic motion such as the change of direction. The thermodiffusion is very sensitive to the molecular interaction between solute and solvent molecules. Therefore a study of the thermophoretic behavior of a group of solute molecules, systematically

\footnotetext{
a)Electronic mail: rkita@keyaki.cc.u-tokai.ac.jp
}

varied in their chemical structure, will help to understand the molecular mechanisms of the thermodiffusion and become a guide in applications.

The thermophoretic behavior has been studied experimentally ${ }^{5-13}$, theoretically ${ }^{14-16}$, and by simulations ${ }^{17-21}$. These previous studies indicated that the thermal diffusion depends on many parameters such as charge, interfacial effects, and molecular interactions. For aqueous systems, the charge effect has been studied systematically showing that $S_{\mathrm{T}}$ increases with decreasing ionic strength. Especially, aqueous mixtures show often a negative $S_{\mathrm{T}}$ at low temperatures. The temperature and concentration dependences of $S_{\mathrm{T}}$ have been studied under various conditions, such as close to the critical micelle concentration and in microemulsions ${ }^{22-24}$. It is considered that the value of $S_{\mathrm{T}}$ of the solute increases if the hydrogen bond network breaks. ${ }^{25}$. There are two mechanisms which can lead to a breaking of hydrogen bonds between solute and water molecules. One possibility is to add an ingredient with a stronger affinity to water, so that the bonds open ${ }^{9}$. Alternatively, an increase of the temperature disrupts the hydrogen bonds between water and the solute. This leads for many systems to a similar temperature dependence of $S_{\mathrm{T}}$ for aqueous solutions of biological and synthetic molecules ${ }^{26}$, which can be described by an empirical equation proposed by Iacopini and Piazza ${ }^{27}$. One might argue qualitatively that the system tries to minimize its local free energy by forming hydrogen bonds at a low overall temperature so that the water molecules accumulate at the cold side. While at a higher overall temperature the system minimizes its free energy by maximizing the orientational and translational entropy $^{13}$.

In this study, we study the contributions of the molec- 
ular weight and hydrogen bonds on the temperature dependence of the Ludwig-Soret effect for aqueous solutions of ethylene glycol oligomers (EGOs), crown ethers (CEs), and glycerol by Thermal Diffusion Forced Rayleigh Scattering (TDFRS). The thermal diffusion of aqueous solutions of EGOs and poly(ethylene glycol)s has been studied experimentally by TDFRS and by the thermogravitational column method, ${ }^{5-7,14,28-30}$. It is the only water soluble polymer for which the molecular weight dependence has been investigated systematically. Additionally it is an interesting system to study the effect of hydrogen bonds on the thermal diffusion behavior. By changing the number of repeating unit of EGOs and CEs, we can control the numbers of donors and acceptors for hydrogen bonds systematically. In order to avoid interactions between solute molecules we studied all solutions at a concentration of $1 \mathrm{wt} \%$. This corresponds approximately to a volume fraction of the order of $1 \%$ and lies well below the overlap concentration. The solute molecules used in this study are listed in Table I. Figure 1 shows the chemical structures of representative compounds, which have been investigated in water. Glycerol has three hydroxyl groups the largest number of donors and no acceptor. All EGOs have two donors and depending on their length also ether groups in their chain which act as acceptor. CEs form a cyclic structure with ether groups in the ring, so that CEs have no donors.

\section{EXPERIMENTAL}

Investigated substances were ethylene glycol (EG, Wako chemicals, $\geq 99.5 \%$ purity), diethylene glycol (2EG, Wako chemicals, $\geq 99 \%$ ), triethylene glycol (3EG, TCI, $\geq 99 \%$ ), tetraethylene glycol (4EG, Wako chemicals, $\geq 99 \%$ ), pentaethylene glycol (5EG, Wako chemicals, $\geq 99 \%$ ), hexaethylene glycol (6EG, Wako chemicals, $\geq 99 \%$ ), dioxane (we call it as CE2, Wako chemicals, $\geq 99.5 \%$ ), 12-crown-4 ether (CE4, TCI, $\geq 95 \%$ ), 15-crown-5 ether (CE5, TCI, $\geq 97 \%$ ), 18-crown-6 ether (CE6, TCI, $\geq 98 \%$ ), and glycerol (Wako chemicals, $\geq$ $99 \%$ ). All of them were dissolved in distilled and deionized water (milli-Q) with a concentration of $1.0 \mathrm{wt} \%$. A small amount of Basantol gelb 215 (BASF) was added to all samples to achieve a sufficient absorption of the writing beam. It is a trivalent salt soluble in water with a strong absorption around $480 \mathrm{~nm} .^{31}$

The concentration part of the normalized heterodyne signal, $\zeta_{\text {het }}$, of the TDFRS experiments is given by ${ }^{32}$

$$
\zeta_{\text {het }}(t)=1-A\left[1-\exp \left(-\frac{t}{\tau}\right)\right]
$$

where $\tau$ is the time constant of the collective diffusion and the amplitude $A$ of concentration signal owing to the Ludwig-Soret effect is given by

$$
A=\frac{(\partial n / \partial w)_{p, T}}{(\partial n / \partial T)_{p, w}} S_{\mathrm{T}} w(1-w) .
$$
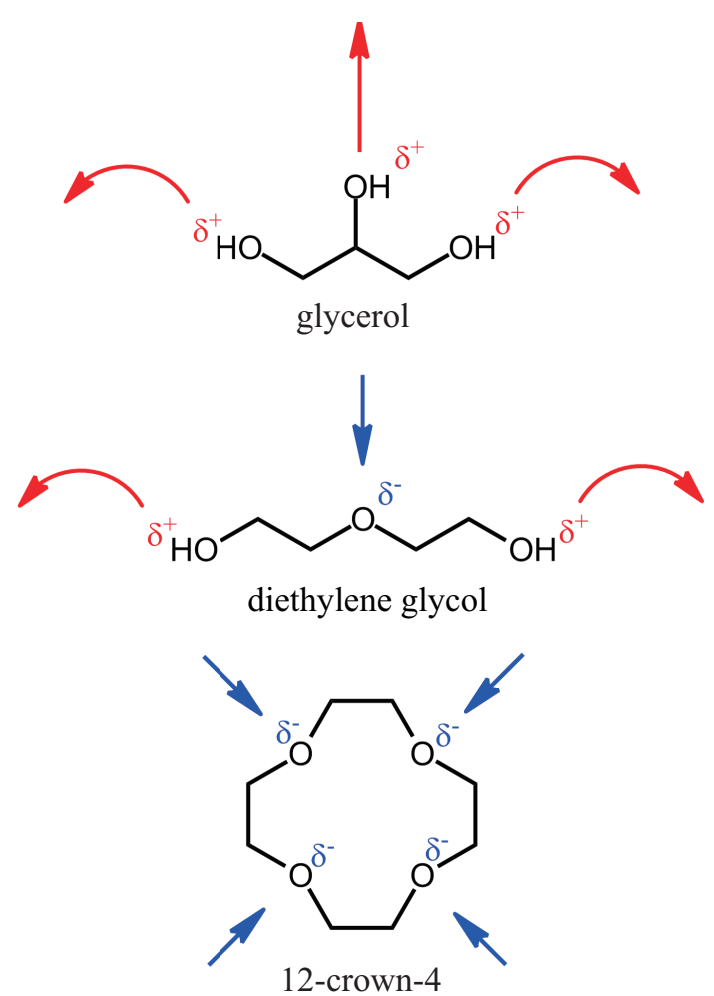

FIG. 1. The chemical structures of representative compounds of this study, Glycerol has with three hydroxyl groups the largest number of donors and no acceptor. Diethylene glycol has beside two donors also one acceptor. The 12-crown-4 ethers has four acceptors.

The refractive index with respect to temperature and concentration $(\partial n / \partial T)_{p, w}$ and $(\partial n / \partial w)_{p, T}$ were measured by a Michelson interferometer ${ }^{33}$ and an Abbe refractometer (DR-M2, ATAGO). Figure 2 shows a typical TDFRS signal for the aqueous solution of CE5 at different temperatures. The fast process describes the heat diffusion and the slow process corresponds to the mass diffusion. From the time constant and the amplitude of the slow process, the mutual diffusion coefficient, $D$, and the Soret coefficient, $S_{\mathrm{T}}$, were obtained by a least-square fit. We performed temperature dependent measurements in the range between 13.7 and $65.7 \pm 0.2{ }^{\circ} \mathrm{C}$.

\section{RESULTS AND DISCUSSION}

Figure 3 (a) and (b) show the Soret coefficient, $S_{\mathrm{T}}$, as a function of temperature for ethylene glycol oligomers (EGOs), crown ethers (CEs), and glycerol in water. The Soret coefficient, $S_{\mathrm{T}}$, shows a positive sign in the investigated temperature range, and $S_{\mathrm{T}}$ increases with increasing molecular weight of EGOs and CEs. In the studied temperature range the Soret coefficients of all systems show a linear trend with respect to temperature. The slight systematic deviations occurring for some systems 
TABLE I. The numbers of the donor $N_{\text {don }}$ and acceptor $N_{\text {acc }}$ for the studied solutes, and the obtained parameters $S_{\mathrm{T} 0}$ and $S_{\mathrm{T} 1}$ in Eq. (4), and $D_{\mathrm{T} 0}$ and $D_{\mathrm{T} 1}$ in Eq. (6).

\begin{tabular}{|c|c|c|c|c|c|c|}
\hline Sample & $N_{\text {don }}$ & $N_{\text {acc }}$ & $\begin{array}{l}S_{\mathrm{T} 0} \\
/ 10^{-3} \mathrm{~K}^{-1}\end{array}$ & $\begin{array}{l}S_{\mathrm{T} 1} \\
/ 10^{-5} \mathrm{~K}^{-2}\end{array}$ & $\begin{array}{l}D_{\mathrm{T} 0} \\
/ 10^{-12} \mathrm{~m}^{2} \mathrm{~s}^{-1} \mathrm{~K}^{-1}\end{array}$ & $\begin{array}{l}D_{\mathrm{T} 1} \\
/ 10^{-14} \mathrm{~m}^{2} \mathrm{~s}^{-1} \mathrm{~K}^{-2}\end{array}$ \\
\hline EG (ethylene glycol) & 2 & 0 & $4.4 \pm 0.4$ & $1.1 \pm 1.1$ & $1.7 \pm 0.7$ & $7.5 \pm 2.0$ \\
\hline 2EG (diethylene glycol) & 2 & 1 & $7.5 \pm 0.2$ & $-2.6 \pm 0.5$ & $2.8 \pm 0.6$ & $7.9 \pm 1.6$ \\
\hline 3EG (triethylene glycol) & 2 & 2 & $9.4 \pm 0.6$ & $-3.0 \pm 1.6$ & $3.1 \pm 0.9$ & $11.8 \pm 2.3$ \\
\hline 4EG (tetraethylene glycol) & 2 & 3 & $10.1 \pm 0.6$ & $-1.3 \pm 1.6$ & $2.6 \pm 0.5$ & $14.4 \pm 1.2$ \\
\hline 5EG (pentaethylene glycol) & 2 & 4 & $12.5 \pm 0.5$ & $-5.3 \pm 1.2$ & $2.4 \pm 1.2$ & $13.6 \pm 2.8$ \\
\hline 6EG (hexaethylene glycol) & 2 & 5 & $13.6 \pm 0.6$ & $-5.2 \pm 1.4$ & $3.5 \pm 0.3$ & $13.0 \pm 0.6$ \\
\hline CE2 (dioxane) & 0 & 2 & $9.6 \pm 0.2$ & $-3.4 \pm 0.5$ & $4.8 \pm 0.3$ & $12.3 \pm 0.9$ \\
\hline CE4 (12-crown-4 ether) & 0 & 4 & $13.2 \pm 0.9$ & $-4.0 \pm 2.2$ & $5.5 \pm 1.8$ & $13.9 \pm 4.4$ \\
\hline CE5 (15-crown-5 ether) & 0 & 5 & $15.5 \pm 0.3$ & $-5.4 \pm 0.8$ & $5.1 \pm 0.7$ & $16.8 \pm 1.8$ \\
\hline CE6 (18-crown-6 ether) & 0 & 6 & $17.4 \pm 1.2$ & $-6.0 \pm 2.6$ & $5.3 \pm 2.4$ & $17.4 \pm 5.5$ \\
\hline glycerol & 3 & 0 & $4.1 \pm 0.2$ & $3.9 \pm 0.5$ & $1.4 \pm 0.3$ & $9.3 \pm 0.9$ \\
\hline
\end{tabular}

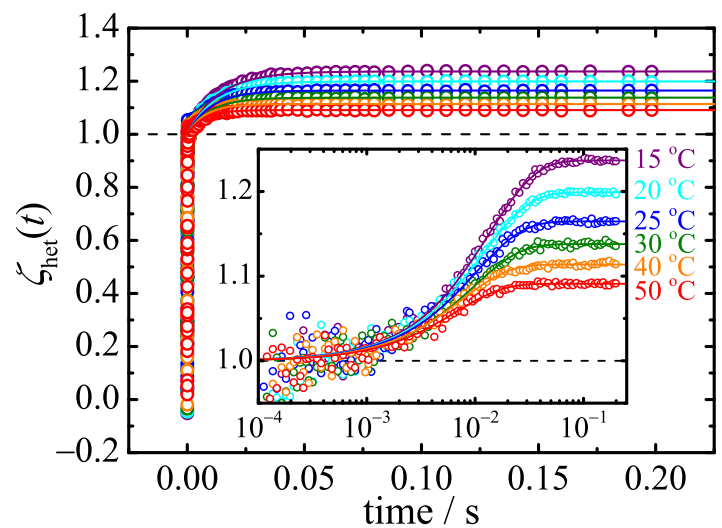

FIG. 2. Typical results of the normalized heterodyne signal of TDFRS measurement for $1.0 \mathrm{wt} \%$ aqueous solution of $15-$ crown-5 ether measured at several temperatures.

(e.g. tetraethylene glycol and hexaethylene glycol) are within the typical uncertainties of $5-10 \%$. The temperature dependence of $S_{\mathrm{T}}$ can be described by

$$
S_{\mathrm{T}}(T)=S_{\mathrm{T} 0}+S_{\mathrm{T} 1} T
$$

Here, $S_{\mathrm{T} 0}$ and $S_{\mathrm{T} 1}$ are parameters corresponding to the intercept and slope, respectively. The obtained parameters are listed in Table I. The slope $S_{\mathrm{T} 1}$ is positive for ethylene glycol (EG) and glycerol, while it is negative for all other substances. The decay of $S_{\mathrm{T}}$ with increasing temperature is unusual, but it has been observed for low concentrations of ethanol and dimethyl sulfoxide in water ${ }^{34}$. The temperature dependence in the investigated range is fully described by a linear expression so that it is not necessary to use the proposed empirical equation,

$$
S_{\mathrm{T}}(T)=S_{\mathrm{T}}^{\infty}\left[1-\exp \left(\frac{T^{*}-T}{T_{0}}\right)\right],
$$

by Iacopini and Piazza ${ }^{27}$, which accounts also for a curvature. Using this simple approach (cf. Eq. 4) we have only two instead of three adjustable parameters. This linear relation observed here may be related to the smaller number of hydroxyl groups or a weaker hydrogen bond capability in comparison with other aqueous systems of SDS micelles, proteins, DNA, and polysaccharides ${ }^{12,13,26,35,36}$. Additionally note that for many aqueous systems, which are described by Eq. 5, the temperature dependence of $S_{\mathrm{T}}$ of the solute molecules in water is more pronounced at low temperatures, because the influence of the formation of hydrogen bonds on the free energy of the system is larger, while at higher temperatures the entropic contributions dominate.

Figure 3 (c) shows the thermal diffusion coefficient, $D_{\mathrm{T}}$, as a function of temperature. The thermal diffusion coefficient can also be described by a linear regression for all systems in the investigated temperature range, although the data points scatter due to experimental difficulties caused by a low solute concentration. The thermal diffusion coefficient, $D_{\mathrm{T}}$, is fitted by the following equation,

$$
D_{\mathrm{T}}(T)=D_{\mathrm{T} 0}+D_{\mathrm{T} 1} T .
$$

The intercept $D_{\mathrm{T} 0}$ and the slope $D_{\mathrm{T} 1}$ are determined by a linear fit. The magnitude of $D_{\mathrm{T}}(T)$ for CEs is larger than that of EGOs and increases with increasing number of repeat unit for EGOs and CEs. The same trend is observed for the Soret coefficient $S_{\mathrm{T}}$. In many aqueous systems, the thermal diffusion coefficient $D_{\mathrm{T}}$ increases with increasing temperature and shows for some systems a sign change. Often a linear relation between $D_{\mathrm{T}}$ and 

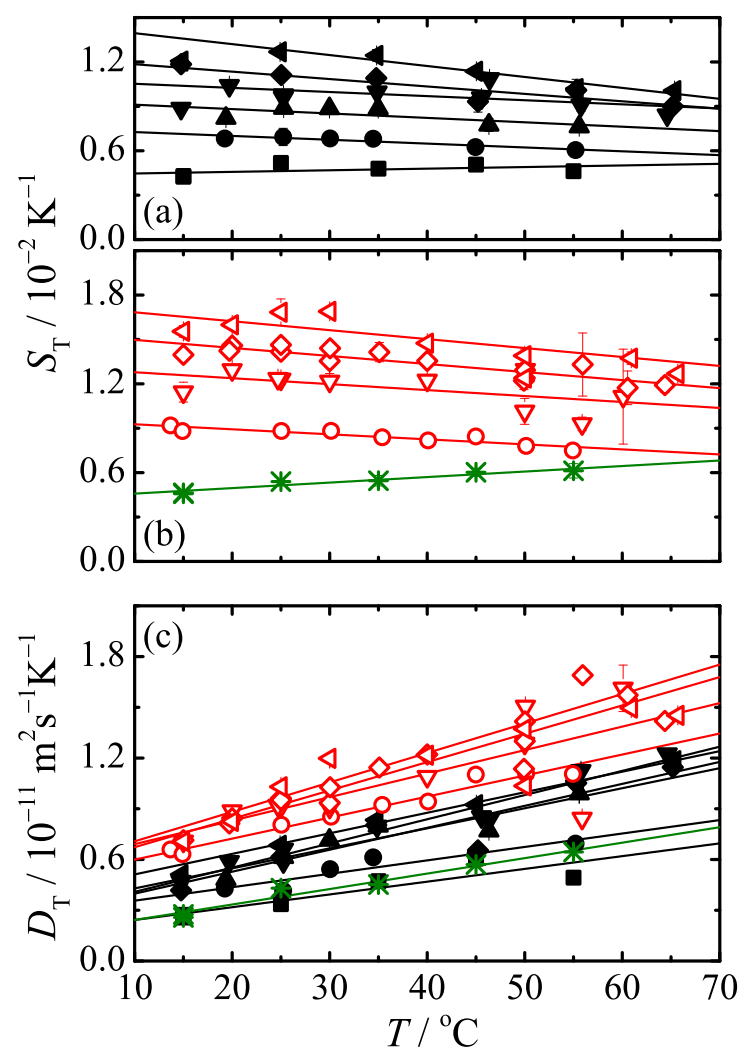

FIG. 3. Soret and thermal diffusion coefficients as function of temperature for $1.0 \mathrm{wt} \%$ aqueous solutions of ethylene glycol $(\mathbf{\square})$, diethylene glycol $(\bullet)$, triethylene glycol $(\boldsymbol{\Delta})$, tetraethylene glycol $(\mathbf{\nabla})$, pentaethylene glycol $(\checkmark)$, hexaethylene glycol $(\triangleleft)$, dioxane $(\circ)$, 12-crown-4 ether $(\nabla), 15$-crown-5 ether $(\diamond)$, 18 -crown-6 ether $(\triangleleft)$, and glycerol $(*)$.

temperature has been observed, although there is an exception for oligo- and poly- saccharide systems where a curvature of $D_{\mathrm{T}}(T)$ is observed on the plot of $D_{\mathrm{T}}(T)$ versus temperature if solute molecules with different molecular weights are considered ${ }^{12}$.

The mutual diffusion coefficient, $D$, as a function of temperature for EGOs, CEs, and glycerol in water is shown in Fig. S1. ${ }^{37}$ In the studied temperature range the mutual diffusion coefficient $D$ of all systems increases linearly with increasing temperature owing to a decrease of the viscosity. Furthermore $D$ decreases with increasing molecular weight.

Figures 4 shows the obtained parameters $S_{\mathrm{T} 0}$ and $S_{\mathrm{T} 1}$ in Eq. (4) as a function of the molecular weight of EGOs (filled circles), CEs (open triangles), and glycerol (asterisk). The intercept $S_{\mathrm{T} 0}$ increases and the slope $S_{\mathrm{T} 1}$ decreases linearly with increasing molecular weight of EGOs and CEs. Chan et al. showed the experimental results for $S_{\mathrm{T}}$ of ethylene glycols in water at $25{ }^{\circ} \mathrm{C}$ in a broad molecular weight range, where they showed $S_{\mathrm{T}}$ can be described by a power law $S_{\mathrm{T}} \propto M^{\nu}$ with $\nu=0.53$ and $M$ the molecular weight of the solute molecule. ${ }^{6}$ These

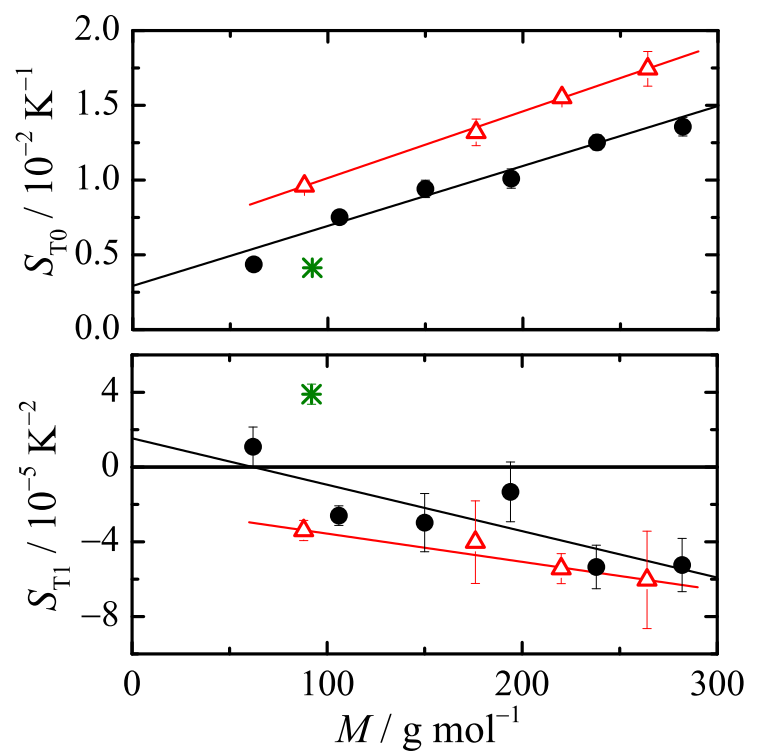

FIG. 4. The intercept $S_{\mathrm{T} 0}$ and slope $S_{\mathrm{T} 1}$ as function of molecular weight for ethylene glycol oligomers $(\bullet)$, crown ethers $(\triangle)$, and glycerol $(*)$.

results agree with findings by Stadelmaier and Köhler for styrene oligomers and polymers in non-polar various solvents. They find values of $\nu$ between 0.5 and $0.6 .^{38}$ The systems used in our study are in the oligomer range and we tentatively show the double-logarithmic plot of $S_{\mathrm{T}}$ vs. $M$ to elucidate the exponent $\nu$. In Fig 5 we plot the intercept $S_{\mathrm{T} 0}$ to eliminate the temperature effect. The lines are obtained by a least-square fit and the obtained exponents are $\nu=0.65 \pm 0.05$ for EGOs and $\nu=0.52 \pm$ 0.02 for CEs. These results suggest that the power law relation is still appropriate for oligomers so that intercept $S_{\text {T0 }}$ can be expressed by

$$
S_{\mathrm{T} 0}(M)=\alpha M^{\nu}
$$

where $\alpha$ is a system dependent parameter.

Figure 6 shows the intercept $D_{\mathrm{T} 0}$ and the slope $D_{\mathrm{T} 1}$ according to Eq. (6) of EGOs, CEs, and glycerol as function of molecular weight. The intercept $D_{\mathrm{T} 0}$ is almost flat and slope $D_{\mathrm{T} 1}$ increases with increasing molecular weight. The thermal diffusion coefficient, $D_{\mathrm{T}}$, of polystyrene in various organic solvents has been studied as a function of molecular weight by Stadelmaier and Köhler ${ }^{38}$. It turned out that the thermal diffusion coefficient, $D_{\mathrm{T}}$, does not depend on the molecular weight for polymers, while it shows a molecular weight dependence for oligomers. This tendency is expressed as $D_{\mathrm{T}}=D_{\mathrm{T}}^{\infty}-a_{1} / M$. In our study the molecular weight range is too narrow compared to the PS study and the intercept $D_{\mathrm{T} 0}$ is nearly flat with respect to molecular weight. We assume that the intercept $D_{\mathrm{T} 0}$ does not depend on the molecular weight, which can be expressed as, 


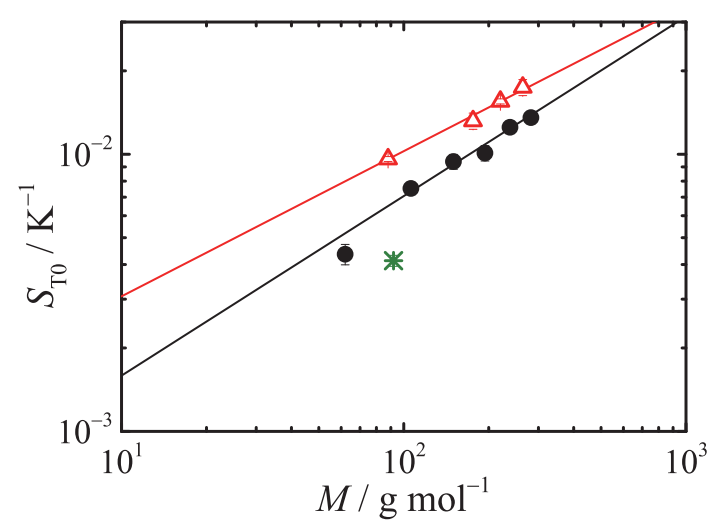

FIG. 5. Double logarithmic plot of the intercept $S_{\mathrm{T} 0}$ as a function of the molecular weight $M$ for ethylene glycol oligomers $(\bullet)$, crown ethers $(\triangle)$, and glycerol $(*)$. Lines are drawn by a least-square fit using Eq. (7).

$$
D_{\mathrm{T} 0}=\alpha^{\prime} \text { (const.). }
$$

Note that without loosing generality we selected $D_{\text {T0 }}$, the thermal diffusion coefficient at $T=0{ }^{\circ} \mathrm{C}$, but we could have selected the thermal diffusion coefficient at any other temperature.
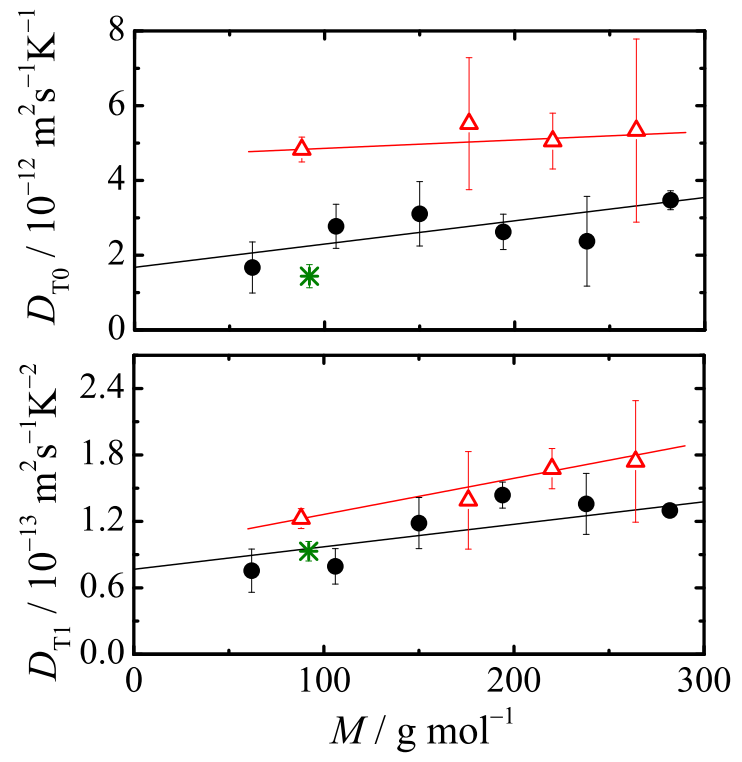

FIG. 6. Intercept $D_{\mathrm{T} 0}$ and slope $D_{\mathrm{T} 1}$ as function of molecular weight for ethylene glycol oligomers $(\bullet)$, crown ethers $(\triangle)$, and glycerol $(*)$.

Previous studies showed that the hydrophobic/hydrophilic balance influences the Ludwig-Soret effect and is related to the capability to form hydrogen bonds in aqueous systems. In this study, we try to correlate the thermophoretic behavior with the capability to form hydrogen bonds in a quantitative manner by introducing the number of donors and acceptors. As mentioned in the introduction hydroxyl groups can work as a donor for hydrogen bonds and ether groups can act as an acceptor for hydrogen bonds (cf. Fig. 1). The strength of hydrogen bonds capability can be systematically varied by the number of repeating units of EGOs and CEs. The number of hydroxyl and ether groups are listed in Table I. We propose the following expression to describe the Soret coefficient with respect to the number of donor, $N_{\text {don }}$, and of acceptor, $N_{\text {acc }}$, groups, respectively,

$$
S_{\mathrm{T}}\left(N_{\mathrm{acc}}, N_{\mathrm{don}}\right)=S_{\mathrm{T}}^{\Delta}+\beta_{0}\left(N_{\mathrm{don}}-N_{\mathrm{acc}}\right) .
$$

Here, $S_{\mathrm{T}}\left(N_{\mathrm{acc}}, N_{\mathrm{don}}\right)$ is the value of $S_{\mathrm{T}}$ at $25{ }^{\circ} \mathrm{C} . S_{\mathrm{T}}^{\Delta}$ is independent term from the hydrogen bonding capability of the solute, while $\beta_{0}$ describes the strength of the hydrogen bond effect for Soret coefficient due to the difference of $N_{\text {don }}$ and $N_{\text {acc }}$. When we plot the $S_{\mathrm{T}}-S_{\mathrm{T}}^{\Delta}$ as a function of $N_{\text {don }}-N_{\text {acc }}$, it gives one master line for all systems with $S_{\mathrm{T}}^{\Delta}=(8.1 \pm 0.3) \times 10^{-3} \mathrm{~K}^{-1}$ and $\beta_{0}=$ $-0.0012 \mathrm{~K}^{-1}$ as shown in Fig. 7 (a). Note that CEs have no hydroxyl groups which can work only as acceptor for hydrogen bonds, while EGOs have donor and acceptor groups. Glycerol has only acceptor groups. The result indicates that the balance of donor and acceptor groups contributes to the magnitude of $S_{\mathrm{T}}$ significantly and Eq. (9) can be utilized for describing the hydrogen bond effect of the investigated systems.

Figure 7 (b) shows the slope $S_{\mathrm{T} 1}$ versus $N_{\text {don }}-N_{\text {acc }}$ which is described by the following equation,

$$
S_{\mathrm{T} 1}\left(N_{\mathrm{don}}, N_{\mathrm{acc}}\right)=\beta\left(N_{\mathrm{don}}-N_{\mathrm{acc}}\right),
$$

where $\beta$ is a parameter obtained as $\beta=(1.2 \pm 0.3) \times$ $10^{-5} \mathrm{~K}^{-2}$. This relation suggests that the temperature dependence of $S_{\mathrm{T}}$ is also characterized by the difference of the numbers of donor and acceptor in the solute molecule.

Furthermore, in order to describe the Soret coefficient we tentatively combine the temperature effect, the molecular weight, and the number of donor and acceptor sites using Eqs. (4), (7), and (10) as follows,

$$
S_{\mathrm{T}}\left(M, T, N_{\mathrm{don}}, N_{\mathrm{acc}}\right)=\alpha M^{\nu}+\beta\left(N_{\mathrm{don}}-N_{\mathrm{acc}}\right) T .
$$

In this empirical approach the Soret coefficient, $S_{\mathrm{T}}$, can be described by contributions related to molecular weight, temperature, and number difference of donor and acceptor sites for hydrogen bonds.

Analog with Eq. (9), we analyze the thermal diffusion coefficient, $D_{\mathrm{T}}$ assuming a proportionality to the number difference of donor and acceptor sites for hydrogen bonds as follows,

$$
D_{\mathrm{T}}\left(N_{\mathrm{don}}, N_{\mathrm{acc}}\right)=D_{\mathrm{T}}^{\Delta}+\beta_{0}^{\prime}\left(N_{\mathrm{don}}-N_{\mathrm{acc}}\right) .
$$




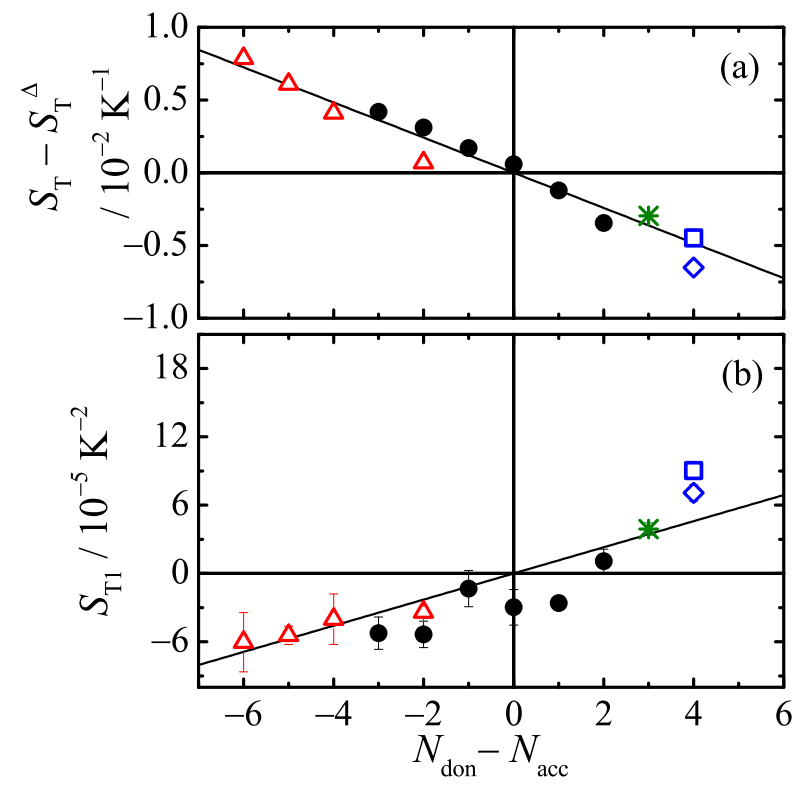

FIG. 7. $S_{\mathrm{T}}-S_{\mathrm{T}}^{\Delta}$ and the slope $S_{\mathrm{T} 1}$ as function of $N_{\mathrm{don}}-$ $N_{\text {acc }}$ for ethylene glycol oligomers $(\bullet)$, crown ethers $(\triangle)$, and glycerol $(*)$. Straight lines in (a) and (b) are obtained by a least-square fit using Eq. (9) and Eq. (10), respectively. Square $(\square)$ and diamond $(\diamond)$ refer to the solutions of glucose in water as their concentrations of $1.0 \mathrm{wt} \%$ [Ref. 12] and 20 wt\% [Ref. 39].

Here, $D_{\mathrm{T}}^{\Delta}$ is the fitting parameter independent of the effect of hydrogen bonds and $\beta_{0}^{\prime}$ is system dependent parameter. As shown in Fig. 8 (a) one master line is obtained, describing $D_{\mathrm{T}}-D_{\mathrm{T}}^{\Delta}$ as function of $N_{\text {don }}-N_{\text {acc }}$ for all systems of EGOs (filled circle), CEs (open triangle), and glycerol (asterisk). Here, the $D_{\mathrm{T}}\left(N_{\mathrm{don}}, N_{\mathrm{acc}}\right)$ value is used at $25{ }^{\circ} \mathrm{C}$. The values $D_{\mathrm{T}}^{\Delta}$ and $\beta_{0}^{\prime}$ are obtained by a least-square fit as $(5.6 \pm 0.3) \times 10^{-12} \mathrm{~m}^{2} \mathrm{~s}^{-1} \mathrm{~K}^{-1}$ and $(-7.5 \pm 0.7) \times 10^{-13} \mathrm{~m}^{2} \mathrm{~s}^{-1} \mathrm{~K}^{-1}$, respectively. For the here studied systems the empirical equation (Eq. 12) is confirmed. The slope of the temperature dependence $D_{\mathrm{T} 1}$ shows also a linear dependence on the difference of donor and acceptor sites for hydrogen bonds and can be expressed by the following linear equation,

$$
D_{\mathrm{T} 1}\left(N_{\mathrm{don}}, N_{\mathrm{acc}}\right)=\beta^{\prime}\left(N_{\mathrm{don}}-N_{\mathrm{acc}}\right)+\gamma^{\prime} .
$$

The fitted straight line in Fig. 8 corresponds to the obtained parameters $\beta^{\prime}$ and $\gamma^{\prime}$ as $(-1.0 \pm 0.2) \times$ $10^{-14} \mathrm{~m}^{2} \mathrm{~s}^{-1} \mathrm{~K}^{-2}$ and $(1.08 \pm 0.05) \times 10^{-13} \mathrm{~m}^{2} \mathrm{~s}^{-1} \mathrm{~K}^{-2}$, respectively. Finally, the temperature, molecular weight, and hydrogen bonds dependences of the thermal diffusion coefficient, $D_{\mathrm{T}}$, can be described by a combination of Eqs. (6), (8), and (13) as follows,

$$
\begin{aligned}
D_{\mathrm{T}}(T, M, & \left.N_{\mathrm{don}}, N_{\mathrm{acc}}\right)=\alpha^{\prime} \\
& +\left[\beta^{\prime}\left(N_{\mathrm{don}}-N_{\mathrm{acc}}\right)+\gamma^{\prime}\right] T .
\end{aligned}
$$

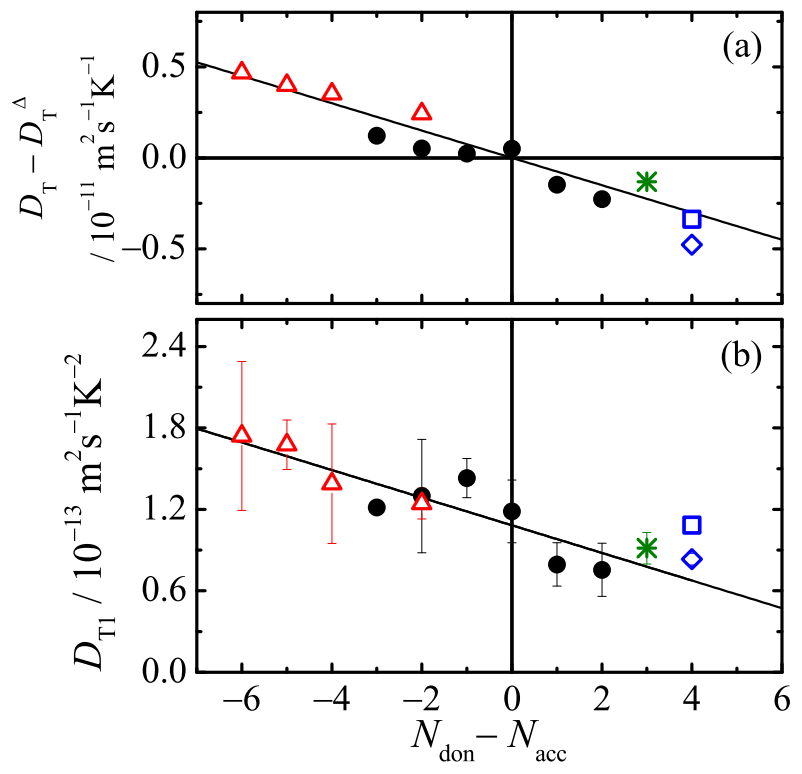

FIG. 8. $D_{\mathrm{T}}-D_{\mathrm{T}}^{\Delta}$ and $D_{\mathrm{T} 1}$ as function of $N_{\text {don }}-N_{\text {acc. }}$ for ethylene glycol oligomers $(\bullet)$, crown ethers $(\triangle)$, and glycerol $(*)$. Straight lines in (a) and (b) are obtained by a least-square fit using Eq. (12) and Eq. (13), respectively. Square( $\square$ ) and diamond $(\diamond)$ refer to the solutions of glucose in water as their concentrations of $1.0 \mathrm{wt} \%$ [Ref. 12] and $20 \mathrm{wt} \%$ [Ref. 39]. All $D_{\mathrm{T}}$ values refer to a temperature of $25^{\circ} \mathrm{C}$.

Equations (11) and (14) express $S_{\mathrm{T}}$ and $D_{\mathrm{T}}$ as function of temperature, molecular weight, and hydrogen bond capability. The empirical expressions indicate that magnitudes of $S_{\mathrm{T}}$ and $D_{\mathrm{T}}$ for the aqueous solutions of EGOs, $\mathrm{CEs}$, and glycerol are described by the sum of a temperature dependent and independent term. The parameters $\alpha$ and $\alpha^{\prime}$ account for the molecular weight effect which are independent from the effects of hydrogen bond capability and temperature. $\beta, \beta^{\prime}$, and $\gamma^{\prime}$ are related to the hydrogen bond capability which can be expressed by the difference of the number of donor and acceptor site of the solute molecules. Here, the hydrogen bond effect can be expressed by the difference of number of donor and acceptor sites $N_{\text {don }}-N_{\text {acc }}$.

According to Eqs. (11) and (14) the Soret and the thermal diffusion coefficient are determined by the product of temperature and the term related to the hydrogen bond capability, $N_{\text {don }}-N_{\text {acc }}$. Depending on the sign of that term we expect an increase or decrease of the coefficient with increasing temperature. The majority of the experiments show that this term is positive. If the dependence on temperature and on the difference $N_{\text {don }}-N_{\text {acc }}$ is linear the fitting can be done in two steps. Alternatively a simultaneous fit on both independent variables can be performed. Using Eqs. (11) and (14) we can reproduce the Soret and thermal diffusion coefficients for EGOs, CEs, and glycerol.

In order to check the applicability of this approach for 
other systems we looked into the system glucose in water and added two points to Figs. 7 and 8. Glucose has five donor and one acceptor sites, which leads to $N_{\text {don }}-N_{\text {acc }}=4$. The Soret and thermal diffusion coefficients for the solution of glucose in water are quoted from literatures corresponding to two different concentrations of $1.0 \mathrm{wt} \%(\square)^{12}$ and $20 \mathrm{wt} \%(\diamond)^{39}$. It turns out that the $S_{\mathrm{T}}-S_{\mathrm{T}}^{\Delta}$ and $D_{\mathrm{T}}-D_{\mathrm{T}}^{\Delta}$ values of glucose in water follow the straight line determined from the measurements of CEs, EGOs, and glycerol in water. The result implies that the difference of donor and acceptor groups for hydrogen bonds is a key parameter to describe the contribution by hydrogen bond effect to the magnitude of $S_{\mathrm{T}}$ and $D_{\mathrm{T}}$ of small water soluble molecules. Applying this concept to larger molecules or macromolecules such as oligo- and poly- saccharides will not be useful, because above a certain number of repeating units the thermal diffusion coefficient will become independent of the molar mass, so that there will be no dependence on the difference of donor and acceptor groups, $N_{\text {don }}-N_{\text {acc }}$.

In summary we showed for aqueous solutions (1.0 wt\%) of ethylene glycol oligomers (from EG to 6EG), crown ethers (from CE2 to CE4), and glycerol, that the Soret coefficient and the thermal diffusion coefficient depend linearly on temperature in the investigated range (13.7 $\left.<T /{ }^{\circ} \mathrm{C}<65.7\right)$. It could be demonstrated that the Soret coefficient and the thermal diffusion coefficient can be separated into three contributions: (1) the molecular weight of the solute, (2) the hydrogen bond effect expressed by the number difference of donor and acceptor sites, and (3) the temperature. The molecular weight effect is independent of temperature, while the temperature dependence of $S_{\mathrm{T}}$ and $D_{\mathrm{T}}$ can be expressed by the product of the hydrogen bond effect and temperature. It is shown that the number of hydrogen bond sites of solute molecules plays a key role for describing the temperature dependence of $S_{\mathrm{T}}$ and $D_{\mathrm{T}}$. Why the coefficients depend on the difference $N_{\text {don }}-N_{\text {acc }}$ is not clear yet and needs further investigation. For future investigations it will be interesting to combine this approach with correlation of the thermophoretic behavior with the octanol-water partition coefficient, $\log p$ [Ref. 13], in order to have a thermophoretic scale as it has been found for non-polar substances ${ }^{40}$.

\section{ACKNOWLEDGMENTS}

This work is partially supported by KAKENHI (No. 26103529 "Fluctuation \& Structure" and No. 24350122) from MEXT, Japan. Additionally, we thank the Deutsche Forschungsgemeinschaft (DFG) for financial support due to Grant No. Wi 1684.

${ }^{1}$ S. de Groot and P. Mazur, Non-equilib. Thermodyn. (New York: Dover, 1984).

${ }^{2}$ C. Ludwig, Sitz. ber. Akad. Wiss. Wien Math.-naturw. Kl 20, 539 (1856).
${ }^{3}$ M. Soret, Arch. Sci. Phys. Nat. 3, 48 (1879).

${ }^{4}$ M. Jerabek-Willemsen, T. André, W. Wanner, H. Roth, S. Duhr, P. Baaske, and D. Breitsprecher, J. Mol. Struct. (2014).

${ }^{5}$ B. de Gans, R. Kita, S. Wiegand, and J. Luettmer-Strathmann, Phys. Rev. Lett. 91, 245501 (2003).

${ }^{6}$ J. Chan, J. Popov, S. Kolisnek-Kehl, and D. Leaist, J. Sol. Chem. 32, 197 (2003).

${ }^{7}$ R. Kita, S. Wiegand, and J. Luettmer-Strathmann, J. Chem. Phys. 121, 3874 (2004).

${ }^{8}$ R. Kita, G. Kircher, and S. Wiegand, J. Chem. Phys. 121, 9140 (2004).

${ }^{9}$ R. Sugaya, B. Wolf, and R. Kita, Biomacromolecules 7, 435 (2006).

${ }^{10}$ H. Ning, J. Buitenhuis, J. Dhont, and S. Wiegand, J. Chem. Phys. 125, 204911 (2006).

${ }^{11}$ P. Blanco and S. Wiegand, J. Phys. Chem. B 114, 2807 (2010).

${ }^{12}$ Y. Kishikawa, H. Shinohara, K. Maeda, Y. Nakamura, S. Wiegand, and R. Kita, Phys. Chem. Chem. Phys. 14, 10147 (2012).

13Z. Wang, H. Kriegs, and S. Wiegand, J. Phys. Chem. B 116, $7463(2012)$

${ }^{14}$ J. Luettmer-Strathmann, J. Chem. Phys. 119, 2892 (2003).

${ }^{15}$ J. Dhont and W. Briels, Euro. Phys. J. E 25, 61 (2008).

${ }^{16}$ A. Würger, Rep. Prog. Phys. 73, 126601 (2010).

${ }^{17}$ D. Reith and F. Müller-Plathe, J. Chem. Phys. 112, 2436 (2000).

${ }^{18}$ B. Rousseau, C. Nieto-Draghi, and J. Avalos, Europhys. Lett. 67, 976 (2004).

${ }^{19}$ P. Polyakov, M. Zhang, F. Müller-Plathe, and S. Wiegand, J. Chem. Phys. 127, 014502 (2007).

${ }^{20}$ P. A. Artola, B. Rousseau, and G. Galliero, J. Am. Chem. Soc. 130, 10963 (2008).

${ }^{21}$ D. Lüsebrink and M. Ripoll, J. Chem. Phys. 137, 194904 (2012).

${ }^{22}$ M. Santos, S. Gomez, E. Bringuier, and A. F. Neto, Phys. Rev. E 77 (2008).

${ }^{23}$ B. Arlt, S. Datta, T. Sottmann, and S. Wiegand, J. Phys. Chem. B 114, 2118 (2010).

${ }^{24}$ P. Naumann, S. Datta, T. Sottmann, B. Arlt, H. Frielinghaus, and S. Wiegand, J . Phys. Chem. B 118, 3451 (2014).

${ }^{25}$ K. Maeda, N. Shinyashiki, S. Yagihara, S. Wiegand, and R. Kita, Euro. Phys. J. E 37, 94 (2014).

${ }^{26}$ Y. Kishikawa, S. Wiegand, and R. Kita, Biomacromolecules 11, 740 (2010).

${ }^{27}$ S. Iacopini and R. Piazza, Europhys. Lett. 63, 247 (2003).

${ }^{28}$ J. Luettmer-Strathmann, Int. J. Thermophys. 26, 1693 (2005).

${ }^{29}$ M. Klein and S. Wiegand, Phys. Chem. Chem. Phys. 13, 7090 (2011).

${ }^{30}$ Z. Wang, D. Afanasenkau, M. Dong, D. Huang, and S. Wiegand, J. Chem. Phys. 141, 064904 (2014).

${ }^{31}$ H. Ning, R. Kita, H. Kriegs, J. Luettmer-Strathmann, and S. Wiegand, J. Phys. Chem. B 110, 10746 (2006).

${ }^{32}$ R. Schafer, A. Becker, and W. Köhler, Int. J. Therm. 20, 1 (1999).

${ }^{33}$ G. Wittko and W. Köhler, Philos. Mag. 83, 1973 (2003).

${ }^{34}$ P. Polyakov and S. Wiegand, J. Chem. Phys. 128, 034505 (2008).

${ }^{35}$ P. Baaske, F. M. Weinert, S. Duhr, K. H. Lemke, M. J. Russell, and D. Braun, Proc. Natl. Acad. Sci. USA 107, 9346 (2007).

${ }^{36}$ S. Iacopini, R. Rusconi, and R. Piazza, Eur. Phys. J. E 19, 59 (2006).

${ }^{37}$ See supplemental material at [URL will be inserted by AIP] for further data regarding to the mutual diffusion coefficient $D$ for $1.0 \mathrm{wt} \%$ aqueous solutions of EGOs, CEs, and glycerol.

${ }^{38}$ D. Stadelmaier and W. Köhler, Macromolecules 41, 6205 (2008).

${ }^{39}$ P. Blanco, H. Kriegs, B. Arlt, and S. Wiegand, J. Phys. Chem. B 114, 10740 (2010).

${ }^{40}$ S. Hartmann, G. Wittko, F. Schock, W. Gross, F. Lindner, W. Köhler, and K. I. Morozov, J. Chem. Phys. 141 (2014). 